\title{
BMP/Coco antagonism as a deterministic factor of metastasis dormancy in lung
}

Jarrod Martinez ${ }^{1}$ and Xiang H-F Zhang ${ }^{* 1-3}$

\begin{abstract}
A recent Cell publication demonstrates that the secreted antagonist of transforming growth factorbeta ligands, Coco, can re-activate previously dormant metastatic breast cancer cells specifically in the lung by inhibiting bone morphogenetic protein (BMP) signaling. The authors provided evidence for a connection between Coco/BMP signaling and molecular and cellular traits of cancer stem cells. Their findings represent a significant advance in our understanding of metastatic dormancy, an extremely important clinical issue that remains understudied. Equally as important, this study also opens interesting avenues for future research.
\end{abstract}

\section{Background}

Metastases account for the vast majority of breast cancerrelated deaths. Recurrence often develops many years after treatment. It is speculated that, for the duration of this latency, disseminated tumor cells exist in distant secondary tissue sites as dormant micrometastases. There is very little information about theses metastatic cancer cells during their dormant states or about how these cells exit from dormancy to re-initiate tumors in distant organs. Hypotheses regarding mechanisms underpinning dormancy include competition between apoptosis and proliferation, due to a lack of a sufficient blood supply [1], and inhibition of tumor growth by the immune system [2]. Molecularly, it was proposed that the competition between the FAK-ERK-mediated mitogenic signals and the p38-mediated stress signals determines the fate of latent cancer cells $[3,4]$. It is known that metastases occur at different frequencies to different

*Correspondence: xiangz@bcm.edu

'Department of Molecular and Cellular Biology, Baylor College of Medicine, One Baylor Plaza, Houston, TX 77030, USA

Full list of author information is available at the end of the article organs and that bone is the most frequent. Cancer stem cells (CSCs) are commonly identified as the culprits of metastatic relapse. This is due in part to the ability of CSCs to self-renew and generate malignant progeny that are usually resistant to chemotherapy and other therapeutic treatments [5,6]. However, how CSC-associated traits are connected to metastasis dormancy remains largely elusive.

\section{The article}

Gao and colleagues [7] set out to address the question of re-activation of latent metastatic breast cancer cells in the lungs of mice. The authors used a cDNA library generated from $4 \mathrm{~T} 1$ cells, a cell line that efficiently colonizes multiple organs, to select for genes that mediate metastases. They identified, among other candidates, Coco, an antagonist of bone morphogenetic protein (BMP) signaling. Overexpression of Coco activates dormant metastatic 4T07 cells, whereas the knockdown of Coco induced the majority of $4 \mathrm{~T} 1$ cells to enter a state of dormancy. Interestingly, the activation of dormant cells is also associated with the enhancement of CSC-associated traits manifested by in vitro mammosphere assays and in vivo tumorigenesis assays. The authors next examined the molecular signaling effectors that are influenced by Coco and validated previous reports that BMP4 stimulates the phosphorylation of Smad proteins in 4T07 cells and that Coco hinders this effect [8]. In line with this finding, BMP signaling seemed to inhibit CSC features. Extensive experiments were also included to verify the roles of Coco and BMP signaling in human cancer cells. To determine the clinical relevance of their finding, the authors derived a 14-gene 'Coco signature' and demonstrated the association with poor prognosis in human microarray datasets. Lastly, the authors provide evidence for the tissue specificity of Coco in lung metastasis dormancy, arguing that endogenous BMP produced by epithelial and mesenchymal cells in the lung suppressed the outgrowth of metastatic seeding of 4T07 cells. Overall, the findings reveal that Coco promotes reactivation from latency by preventing BMP signaling, which in normal cases imposes a dormant state. 


\section{Viewpoint}

This study convincingly demonstrated that Coco can promote the colonization of metastatic breast cancer cells in the lung via the inhibition of the BMP signaling axis. Specific mechanisms involve the re-activation of originally quiescent cancer cells as well as the enhancement of CSC traits, both of which significantly enriched our understanding of metastasis and related biological processes. Moreover, this study raises interesting questions that may warrant further investigations.

First, the lack of Coco renders dormant status to cancer cells. Forced expression of this protein 'woke up' the dormant cells, leading to full-fledged colonization. However, what could be the mechanisms that drive elevated expression of Coco during natural disease progression resulting in distant recurrence? Given that dormant cancer cells do not constantly proliferate, de novoacquired genomic aberrations would be rare and are unlikely to be responsible for such an alteration. The microenvironment factors and epigenetic reprogramming may be more reasonable answers. However, a system in which natural exit of dormancy occurs may be necessary to investigate this question.

Second, lung specificity of Coco provides a nice example of how dormancy mechanisms may rely on the particular microenvironment. Dormancy in other organs needs to be attacked independently. A previous study demonstrates that prostate cancer cells are turned into a reversible senescent state by BMP7 in the bone [9]. BMP2 is also highly enriched in breast cancer bone metastases [10]. These studies raise the possibility that other BMP family members mediate dormancy in bone, another major target organ of breast cancer metastasis. Given that long-latency distant recurrences usually occur to bone, the mechanism underlying bone metastatic dormancy should also be investigated with great urgency.

Last but not least, the authors provided evidence for a model in which the CSC traits can be enriched or suppressed through microenvironment-induced signal transduction events (in this case, suppression of BMP signaling). Although the perturbation of BMP signaling was done primarily through permanent transduction of regulators such as SMAD6 and Coco, the data clearly suggested that BMP activity of the lung microenvironment could transiently or permanently suppress 'CSCness' of the metastatic seeds. Several interesting questions can be asked on the basis of this finding. Does BMP signaling have different effects in CSCs and non-CSCs defined by conventional criteria? If BMP induces differentiation of CSCs, is such differentiation reversible? Are the dormant cells activated by Coco the originally existing CSCs? Answers to these questions will likely further advance our understanding of metastatic dormancy.

\section{Abbreviations}

BMP, bone morphogenetic protein; CSC, cancer stem cell.

Competing interests

The authors declare that they have no competing interests.

\section{Acknowledgments}

$\mathrm{XZ}$ is a McNair Medical Institute Scholar supported by the McNair Medical Institute, R00CA151293 from the National Cancer Institute, and a grant from the Breast Cancer Research Foundation. JM is supported by the graduate program of Molecular and Cellular Biology at the Baylor College of Medicine.

\section{Author details}

'Department of Molecular and Cellular Biology, Baylor College of Medicine, One Baylor Plaza, Houston, TX 77030, USA. 'Lester and Sue Smith Breast Center, Baylor College of Medicine, One Baylor Plaza, Houston, TX 77030, USA. ${ }^{3}$ McNair Medical Institute, 109 N. Post Oak Lane, Suite 600, Houston, TX 77024, USA.

Published: 15 January 2013

\section{References}

1. Gao D, Nolan DJ, Mellick AS, Bambino K, McDonnell K, Mittal V: Endothelial progenitor cells control the angiogenic switch in mouse lung metastasis. Science 2008, 319:195-198.

2. Koebel CM, Vermi W, Swann JB, Zerafa N, Rodig SJ, Old LJ, Smyth MJ, Schreiber RD: Adaptive immunity maintains occult cancer in an equilibrium state. Nature 2007, 450:903-907.

3. Aguirre-Ghiso JA: Models, mechanisms and clinical evidence for cancer dormancy. Nat Rev Cancer 2007, 7:834-846.

4. Sosa MS, Avivar-Valderas A, Bragado P, Wen HC, Aguirre-Ghiso JA: ERK1/2 and p38alpha/beta signaling in tumor cell quiescence: opportunities to control dormant residual disease. Clin Cancer Res 2011, 17:5850-5857.

5. Clevers H: The cancer stem cell: premises, promises and challenges. Nat Med 2011, 17:313-319.

6. Gupta PB, Chaffer CL, Weinberg RA: Cancer stem cells: mirage or reality? Nat Med 2009, 15:1010-1012.

7. Gao H, Chakraborty G, Lee-Lim AP, Mo Q, Decker M, Vonica A, Shen R, Brogi E, Brivanlou AH, Giancotti FG: The BMP inhibitor Coco reactivates breast cancer cells at lung metastatic sites. Cell 2012, 150:764-779.

8. Bell E, Munoz-Sanjuan I, Altmann CR, Vonica A, Brivanlou AH: Cell fate specification and competence by Coco, a maternal BMP, TGFbeta and Wnt inhibitor. Development 2003, 130:1381-1389.

9. Kobayashi A, Okuda H, Xing F, Pandey PR, Watabe M, Hirota S, Pai SK, Liu W, Fukuda K, Chambers C, Wilber A, Watabe K: Bone morphogenetic protein 7 in dormancy and metastasis of prostate cancer stem-like cells in bone. J Exp Med 2011, 208:2641-2655.

10. Zhang XH, Wang Q, Gerald W, Hudis CA, Norton L, Smid M, Foekens JA, Massague J: Latent bone metastasis in breast cancer tied to Src-dependent survival signals. Cancer Cell 2009, 16:67-78.

doi: $10.1186 /$ bcr3350

Cite this article as: Martinez J, Zhang $\mathrm{XH}$ : BMP/Coco antagonism as a deterministic factor of metastasis dormancy in lung. Breast Cancer Research 2013, 15:302 